INPLASY

PROTOCOL

To cite: Chang et al. The effects on pain and disability of traditional Chinese nonpharmacological therapy for knee osteoarthritis: a protocol for systematic review and meta-analysis. Inplasy protocol 202170098. doi:

10.37766/inplasy2021.7.0098

Received: 31 July 2021

Published: 31 July 2021

Corresponding author:

Wei Chang

boyyim@163.com

Author Affiliation:

Community Healthcare Center of Jiangqiao Town.

Support: Jiading Science Project.

Review Stage at time of this submission: Preliminary searches.

Conflicts of interest:

None declared.

\section{The effects on pain and disability of traditional Chinese non- pharmacological therapy for knee osteoarthritis: a protocol for systematic review and meta-analysis}

Chang, W1; Guo, W22.

Review question / Objective: The current systematic review and meta-analysis will be to investigate the effects of traditional Chinese non-pharmacological therapy in the management of KOA. The primary outcomes will be focused on pain and disability of patients with KOA.

Condition being studied: knee osteoarthritis.

Information sources: PubMed, Embase, MEDLINE, Cochrane Central Register of Controlled Trials, Web of Science, China Knowledge Resource Integrated Database, and Wanfang Data from their inception to December 2021.

INPLASY registration number: This protocol was registered with the International Platform of Registered Systematic Review and Meta-Analysis Protocols (INPLASY) on 31 July 2021 and was last updated on 31 July 2021 (registration number INPLASY202170098).

\section{INTRODUCTION}

Review question / Objective: The current systematic review and meta-analysis will be to investigate the effects of traditional Chinese non-pharmacological therapy in the management of KOA. The primary outcomes will be focused on pain and disability of patients with KOA.
Rationale: Knee osteoarthritis (KOA) is a common chronic joint disease characterized by joint pain and functional limitation worldwide. The prevalence of KOA is expected to increase due to increased aging and rates of obesity of the global population. In the United States about 14 million people suffered from pain and disability due to KOA. In Korea, the prevalence of radiographic KOA is $35.1 \%$ 
(24.4\% in men, $44.3 \%$ in women) in adults over $\mathbf{5 0}$ years, and the prevalence is up to $\mathbf{7 8 . 7 \%}$ in women over 80 years. In China, the prevalence rate of $\mathrm{KOA}$ is $8.1 \%$, and the prevalence rate is increasing rapidly among people older than 45 years. According to the Global Burden of Diseases study, the burden of osteoarthritis, especially KOA, is increasing, which will impose new challenges on the health systems, along with mental disorders and diabetes. About the complementary and alternative therapy of KOA, the Osteoarthritis Research Society International (OARSI) and European Society for Clinical and Economic Aspects of Osteoporosis, Osteoarthritis and Musculoskeletal Diseases (ESCEO) both recommend that patient education, exercise, and weight loss (if a patient is overweight) should form the core treatment approach. Non-pharmacological intervention may be an important option for patients with KOA. In China, traditional Chinese non-pharmacological therapies, including acupuncture, Tuina, Tai Chi, etc. are mostly used in the management of KOA. Acupuncture showed beneficial effects in reducing pain due to KOA. Tuina, as a manual therapy, could improve functional limitation of knee joint. The OARSI guidelines also recommend Tai Chi for the first-line treatment of KOA. Traditional Chinese non-pharmacological therapy may be beneficial for limb motor function, balance function, and daily life activity. Currently, the meta-analysis have reported that Tai Chi may be effective for KOA. More and more randomized controlled trials (RCT) have indicated that acupuncture can significantly alleviate pain, relieve stiffness, and improve physical function of patients with KOA. However, the effects of traditional Chinese nonpharmacological therapy for KOA remains controversial. Several trials could not suggest the effects of traditional Chinese non-pharmacological therapy for KOA due to the small sample size, short duration time, etc. Furthermore, previous systematic review and meta-analysis did not focus on the effects of traditional Chinese nonpharmacological therapy for KOA as a whole. In Chinese community hospital, however, acupuncture, Tuina, and Tai Chi are usually in the management of KOA as whole-body treatment.

Condition being studied: knee osteoarthritis.

\section{METHODS}

Search strategy: Two reviewers will independently perform a systematic search of PubMed, Embase, MEDLINE, Cochrane Central Register of Controlled Trials, Web of Science, China Knowledge Resource Integrated Database, and Wanfang Data from their inception to December 2021. The search terms include (1) "knee osteoarthritis"; (2) "acupuncture" or "Tuina" or "Chinese massage" or "moxibustion" or "Taiji" or "Tai Chi" or "Baduanjin" or "Wuqinxi" or "Yijinjing" or "Liuzijue"; (3) "pain" or "disability" or "functional limitation". The search strategy details for PubMed are presented in Table 1. The similar terms will be translated into Chinese for Chinese databases. The language will be limited to English and Chinese.

Participant or population: Participants with diagnosis of KOA by the American College of Rheumatology, the American Rheumatism Association, radio-graphic evidence or physician-confirmed diagnosis. There were no limitations on age, gender, or nationality of patients with KOA.

Intervention: In treatment group, patients with KOA were treated by traditional Chinese non-pharmacological therapy including acupuncture, moxibustion, Tuina, Tai Chi, Baduanjin, Wuqinxi, Liuzijue and Yijinjing, which was used alone or in combination (such as Tuina plus acupuncture).

Comparator: In control group, the patients were treated by medicine, observation, education, sham exercise, sham acupuncture, and so on.

Study designs to be included: RCT of traditional Chinese non-pharmacological therapy in the management of KOA will be included. 
Eligibility criteria: Type of studies. In this meta-analysis, only RCT of traditional Chinese non-pharmacological therapy in the management of KOA will be included. Language will be restricted to English and Chinese. Types of participants. Participants with diagnosis of KOA by the American College of Rheumatology, the American Rheumatism Association, radio-graphic evidence or physician-confirmed diagnosis. There were no limitations on age, gender, or nationality of patients with KOA. Intervention and comparisons. In treatment group, patients with KOA were treated by traditional Chinese non-pharmacological therapy including acupuncture, moxibustion, Tuina, Tai Chi, Baduanjin, Wuqinxi, Liuzijue and Yijinjing, which was used alone or in combination (such as Tuina plus acupuncture). In control group, the patients were treated by medicine, observation, education, sham exercise, sham acupuncture, and so on. Outcomes. Primary outcomes are pain lassessing by visual analogue scale score or any other valuable scales) and disability (assessing by the Western Ontario and McMaster university orthopedic index, Knee Injury and Osteoarthritis Outcome Score, or any other valuable scales). The secondary outcomes will include quality of life (assessing by 36-Item Short Form Survey or any other valuable scales) and adverse events.

Information sources: PubMed, Embase, MEDLINE, Cochrane Central Register of Controlled Trials, Web of Science, China Knowledge Resource Integrated Database, and Wanfang Data from their inception to December 2021.

Main outcome(s): Primary outcomes are pain (assessing by visual analogue scale score or any other valuable scales) and disability (assessing by the Western Ontario and McMaster university orthopedic index, Knee Injury and Osteoarthritis Outcome Score, or any other valuable scales).

Additional outcome(s): The secondary outcomes will include quality of life (assessing by 36 -Item Short Form Survey or any other valuable scales) and adverse events.

Data management: Two reviewers independently extract data from the included articles. The following data will be extracted: reference information (first author, year of publication, etc.), study characteristics (objectives of the study, method of randomization, method of blinding, etc.), participant characteristics (ample size, age, gender, characteristics of KOA, etc.), interventions (type of traditional Chinese non-pharmacological therapy, control interventions, time and frequency of intervention, follow-up time, etc.), outcomes measure, and adverse effects. Any discrepancies will be resolved by discussion between reviewers.

Quality assessment / Risk of bias analysis: The quality of eligible studies will be evaluated by two independent reviewers using the Cochrane Risk of Bias Tool for RCT: Selection bias, performance bias, attribution bias, reporting bias, and other bias, which will be divided in to "low risk of bias", "high risk of bias", or "unclear". Any disagreement will be resolved through discussion.

Strategy of data synthesis: The metaanalysis will be conducted with a random or fixed effect model to calculate the standardized mean difference and $95 \%$ confidence intervals based on different heterogeneity using the Review Manager Version 5.3 software. The test of 12 will be used to identify the heterogeneity. When I2values $>50 \%$, $P$-value $<0.05$, there will be heterogeneity among the studies. The random effect models will be used and the source of heterogeneity should be analyzed. The corresponding authors will be contacted to get detailed information if relevant data are not reported.

Subgroup analysis: The subgroup analysis will be carried based on different types of traditional Chinese non-pharmacological therapy and different outcomes. The patients with KOA will be divided into young ( $<40$ years) and old ( $\geq 40$ years) KOA subgroups according to the age. 
Sensitivity analysis: Sensitivity analysis will be used to assess the reliability of the combined results of meta-analysis for each outcome index.

Language: English and Chinese.

Country(ies) involved: China.

Keywords: pain; disability; traditional

Chinese medicine; knee osteoarthritis.

Contributions of each author:

Author 1 - Wei Chang.

Author 2 - Weina Guo. 